\title{
Resistencia de Shigella spp. a los antimicrobianos en Córdoba, Argentina, durante el período 1990-1997
}

\author{
María Emilia Suárez, ${ }^{1}$ Lydia Carvajal, ${ }^{1}$ Catalina Culasso ${ }^{1}$ \\ y Marisa Paredes ${ }^{1}$
}

RESUMEN En este estudio se analiza la evolución de la resistencia a los antimicrobianos en 771 aislados de Shigella spp. obtenidos de un total de 9195 coprocultivos efectuados entre 1990 y 1997 en un hospital infantil de Córdoba, Argentina. S. flexneri, responsable de $73 \%$ de las infecciones por Shigella, fue la especie más resistente. La frecuencia de la multirresistencia de S. flexneri a los tres antibacterianos más utilizados (ampicilina, trimetoprima-sulfametoxazol y cloranfenicol) aumentó de 10\% en 1990 a 58\% en 1997 (P < 0,001). Cuando se considera separadamente cada uno de ellos, la resistencia a la ampicilina aumentó de 60 a 100\% (P < 0,001), la resistencia al cloranfenicol de 13 a $71 \%(\mathrm{P}<0,001)$ y la resistencia a la trimetoprima-sulfametoxazol de 79 a 84\% (P=0,22). Para S. sonnei, el aumento de la resistencia a la ampicilina (de $36 \%$ en 1990 a 54\% en 1997) no fue estadísticamente significativo ( $\mathrm{P}=0,20)$, ni tampoco lo fue la disminución de la resistencia a la trimetoprima-sulfametoxazol, que pasó de 82\% en 1990 a 55\% en 1997 ( $\mathrm{P}=0,08)$; solo se detectaron dos aislados resistentes al cloranfenicol, uno en 1995 y otro en 1997, y dos con resistencia múltiple a los tres antibióticos. Consideramos obligatorio realizar pruebas de susceptibilidad en cada aislado clínico de Shigella, ya que permiten detectar cambios en el perfil de la resistencia y, con ello, adecuar el tratamiento empírico.

Shigella es uno de los agentes etiológicos más importantes de diarrea aguda en pacientes pediátricos. La mayor tasa de incidencia de la enfermedad se registra en niños de 1 a 4 años de edad, seguidos de los de 5 a 9 años (1). El tratamiento con antibióticos tiende a reducir la duración de la

\footnotetext{
1 Laboratorio de Bacteriología, Hospital de Niños, Córdoba, Argentina. Toda la correspondencia debe ser enviada a María Emilia Suárez a la siguiente dirección postal: Duarte Quirós 310, 5 P “D” (5000). Córdoba. Argentina.
}

fiebre y de la diarrea y las complicaciones letales. Al acortar la duración de la diarrea disminuyen las posibilidades de diseminación del agente (1-3), en la que es muy importante la transmisión de persona a persona y se requiere un inóculo pequeño (10-100 microorganismos) para producir infección.

Este microorganismo, que inicialmente era susceptible a los antimicrobianos más empleados (4), tales como la ampicilina y la trimetoprima-sulfametoxazol (TMP-SMX), desde el inicio de los años 80 comenzó a manifestar resistencia a estos agentes (5-7) en Estados Unidos de América, Canadá,
Tailandia, países africanos y algunos países europeos, como Inglaterra $y$ Gales.

El objetivo de este estudio fue analizar las tendencias actuales de la resistencia de los aislados de Shigella spp. obtenidos por coprocultivo en el Hospital de Niños de Córdoba, Argentina, en el período comprendido entre enero de 1990 y diciembre de 1997.

\section{MATERIALES Y MÉTODOS}

Entre enero de 1990 y diciembre de 1997 se cultivaron 9195 muestras de 
CUADRO 1. Aislados de las diferentes especies de Shigella. Hospital de Niños, Córdoba, Argentina, 1990-1997

\begin{tabular}{|c|c|c|c|c|c|c|c|c|c|c|c|c|c|c|c|c|}
\hline \multirow[b]{3}{*}{ Especie } & \multicolumn{16}{|c|}{ Año } \\
\hline & \multicolumn{2}{|c|}{1990} & \multicolumn{2}{|c|}{1991} & \multicolumn{2}{|c|}{1992} & \multicolumn{2}{|c|}{1993} & \multicolumn{2}{|c|}{1994} & \multicolumn{2}{|c|}{1995} & \multicolumn{2}{|c|}{1996} & \multicolumn{2}{|c|}{1997} \\
\hline & No. & $\%$ & No. & $\%$ & No. & $\%$ & No. & $\%$ & No. & $\%$ & No. & $\%$ & No. & $\%$ & No. & $\%$ \\
\hline \multicolumn{17}{|l|}{ Total de aislados } \\
\hline de Shigella spp. & 55 & $7^{a}$ & 51 & $7^{a}$ & 75 & $10^{\mathrm{a}}$ & 115 & $15^{a}$ & 96 & $12^{a}$ & 112 & $15^{a}$ & 79 & $10^{a}$ & 188 & $24^{a}$ \\
\hline S. flexneri & 38 & 69 & 37 & 73 & 49 & 66 & 58 & 50 & 70 & 73 & 87 & 78 & 55 & 70 & 172 & 91 \\
\hline S. sonnei & 11 & 20 & & & 1 & 1 & 41 & 36 & 16 & 17 & 22 & 20 & 19 & 24 & 11 & 6 \\
\hline S. boydii & & & 1 & 2 & & & 3 & 3 & & & 3 & 2 & 5 & 6 & 5 & 3 \\
\hline No identificada & 6 & 11 & 13 & 25 & 25 & 33 & 13 & 11 & 10 & 10 & & & & & & \\
\hline
\end{tabular}

a Porcentajes de casos aislados en cada año sobre el total de los ocho años.

heces provenientes de 8914 pacientes, de los cuales 5751 eran niños internados y 3163 niños atendidos en las consultas externas del hospital. Se consideró solo un aislamiento positivo por paciente. Las muestras se cultivaron en agar de MacConkey, agar de MacConkey-sorbitol, agar S-S y caldo Selenito de enriquecimiento. La identificación bioquímica de las colonias sospechosas se realizó con métodos estándar (8) y el grupo serológico se identificó por aglutinación con antisueros específicos (División de Antisueros, Instituto de Microbiología “Carlos G. Malbrán").

La susceptibilidad a los antimicrobianos se determinó por el método de difusión con discos, según los procedimientos y criterios de interpretación establecidos por el Comité Nacional de Estándares para Laboratorios Clínicos de los Estados Unidos (The National Committee for Clinical Laboratory Standards: NCCLS) (9). Para el control de calidad se emplearon las cepas Escherichia coli ATCC 25 922, E. coli productora de beta-lactamasa ATCC 35 218,
Staphylococcus aureus ATCC 25 923, Enterococcus faecalis ATCC 29212 y Pseudomonas aeruginosa ATCC 27 853. Con los controles, las dimensiones de las zonas de inhibición alrededor de los discos que contenían los antibióticos ensayados (ampicilina, TMP-SMX, cloranfenicol, gentamicina, colistina $y$ fosfomicina) estuvieron dentro de los límites aceptables según las normas del NCCLS.

El análisis estadístico se realizó con el programa STA. Se aplicó la prueba de la $j i$ al cuadrado $\left(\chi^{2}\right)$ para proporciones y el nivel de significación estadística se fijó en 0,05.

\section{RESULTADOS}

Entre las 9195 muestras procesadas, se obtuvieron 771 aislados de Shigella spp., de los cuales 507 procedieron de pacientes ambulatorios atendidos en consultas externas del hospital, y 264 de pacientes internados. De estos 771 aislados de Shigella spp., 566 (73\%) correspondieron a S. flexneri, 121 (16\%) a
S. sonnei y $17(2 \%)$ a $S$. boydii; los 67 restantes $(9 \%)$ correspondieron a aislados bioquímicamente compatibles con Shigella spp., pero que no fueron sometidos a pruebas serológicas y no se incluyeron en el estudio de resistencia por especie (cuadro 1).

Al considerar todas las especies de Shigella se observó un incremento estadísticamente significativo de la resistencia múltiple a los tres antimicrobianos (ampicilina, TMP-SMX y cloranfenicol) más utilizados en nuestro medio para tratar la shigelosis en pacientes pediátricos: de $7 \%$ en 1990 pasó a $54 \%$ en $1997\left(\alpha_{1 \mathrm{gl}}^{2}=36 ; P<0,001\right)$. Al analizar cada antibiótico por separado, se registró un aumento significativo de la resistencia a la ampicilina (de 64 a $\left.95 \% ; \chi_{1 \mathrm{gl}}^{2}=35 ; P<0,001\right) \mathrm{y}$ al cloranfenicol (de 11 a 62\%; $\chi_{1 \mathrm{gl}}^{2}=43 ; P<$ $0,001)$. En cambio, la resistencia a la TMP-SMX disminuyó, aunque de forma no significativa (de 84 a $78 \%$; $\chi_{1 \mathrm{gl}}^{2}=48 ; P=0,17$ ) (cuadro 2). En cuanto a los otros tres antibióticos, empleados excepcionalmente en el tratamiento de la diarrea infantil, solo se detectaron 3

CUADRO 2. Porcentajes de aislados de Shigella spp. resistentes a la ampicilina, trimetoprima-sulfametoxazol y cloranfenicol. Hospital de Niños, Córdoba, Argentina, 1990-1997

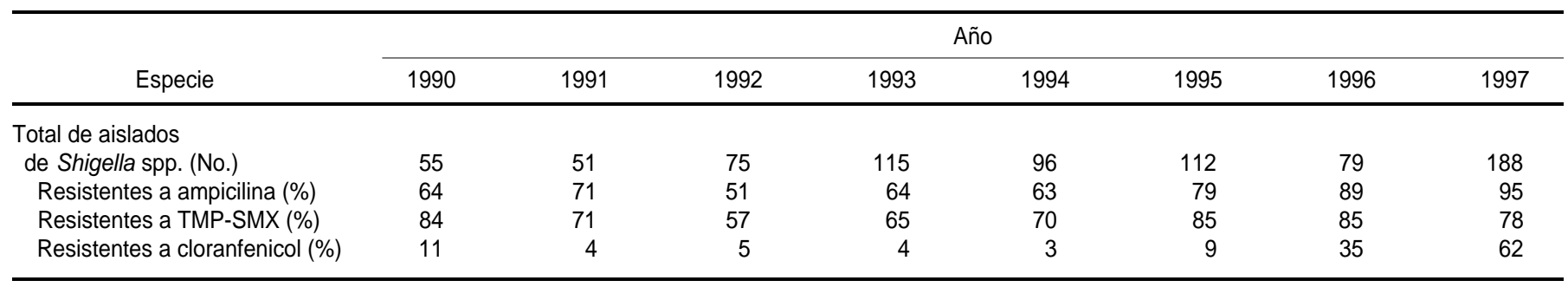


FIGURA 1. Porcentajes de aislados de Shigella flexneri resistentes a la ampicilina, trimetoprima-sulfametoxazol y cloranfenicol. Hospital de Niños, Córdoba, Argentina, 1990-1997

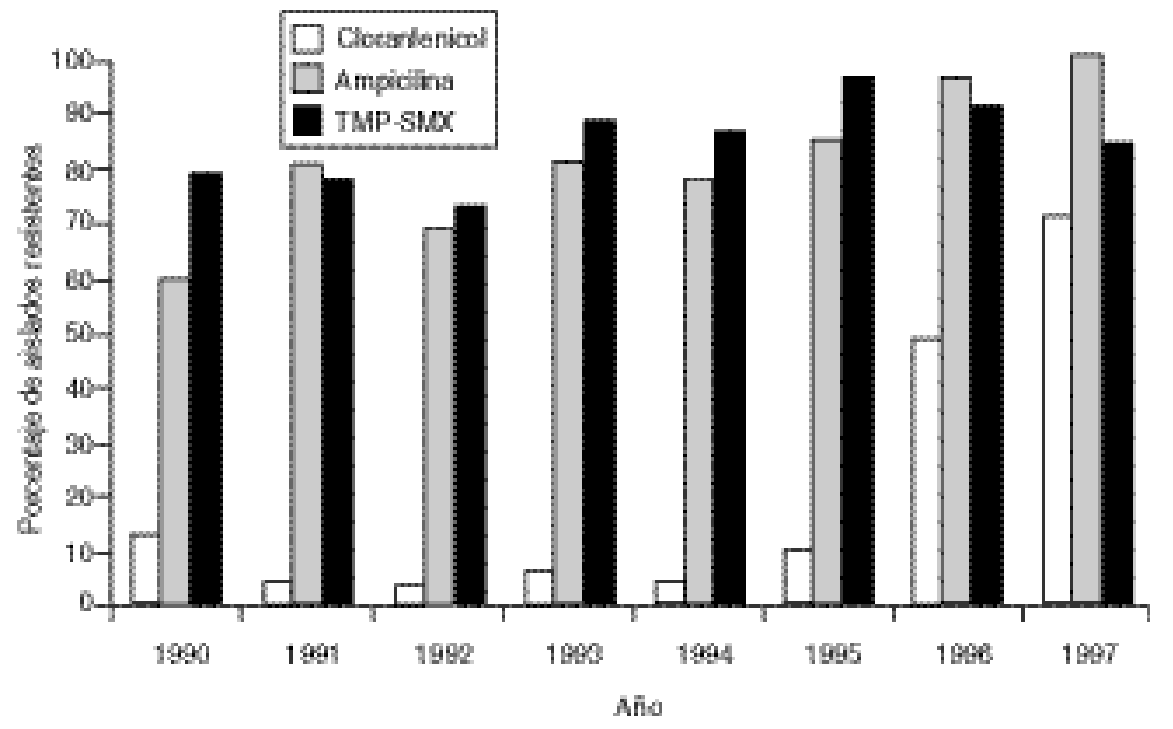

aislados resistentes a la colistina, 5 a la gentamicina y 18 a la fosfomicina.

\section{Evolución de la resistencia en Shigella flexneri}

Considerando cada antibiótico por separado, la resistencia a la ampicilina aumentó de forma significativa (de 60 a $\left.100 \% ; \chi_{1 \mathrm{gl}}^{2}=67 ; P<0,001\right)$, igual que la resistencia al cloranfenicol (de 13 a $\left.71 \% ; \chi_{1 \mathrm{~g} 1}^{2}=42 ; P<0,001\right)$. Para la TMP-SMX el incremento no fue estadísticamente significativo (de 79 a $\left.84 \% ; \chi_{1 \mathrm{gl}}^{2}=0,31 ; P=0,22\right)$, aunque en 1995 y 1996 se alcanzaron cifras de 96 y $91 \%$, respectivamente (figura 1). El aumento de la multirresistencia a estos tres antibióticos también fue estadísti-

FIGURA 2. Porcentajes de aislados de Shigella flexneri que mostraron resistencia múltiple a la ampicilina, trimetoprima-sulfametoxazol y cloranfenicol. Hospital de Niños, Córdoba, Argentina, 1990-1997

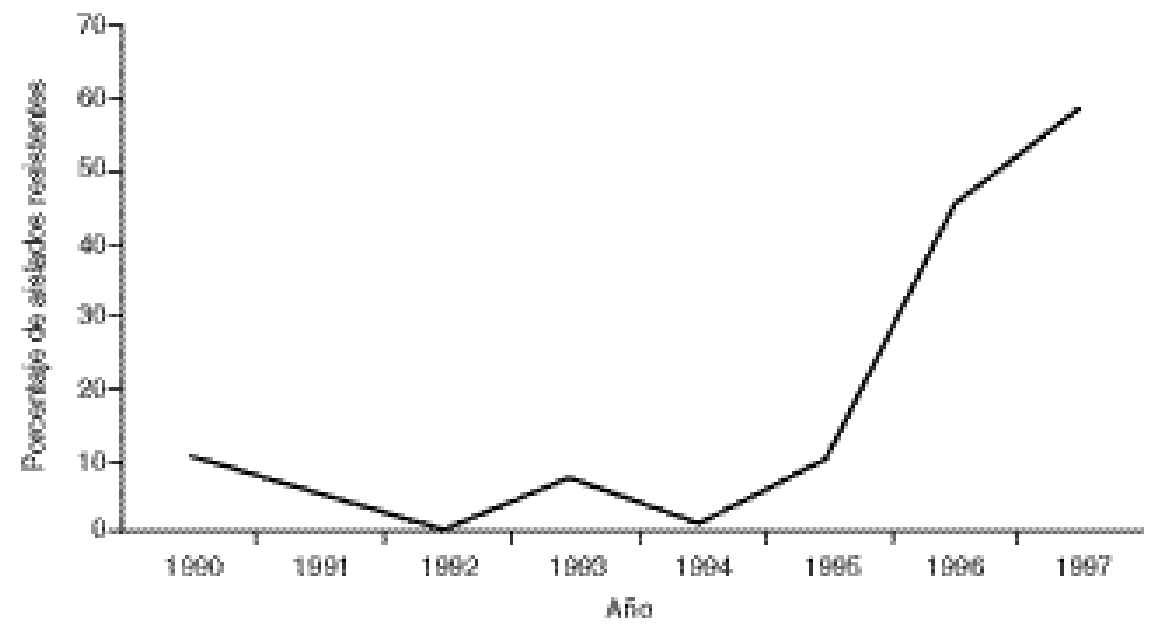

camente significativo $\left(\chi_{1 \mathrm{gl}}^{2}=26 ; P<\right.$ $0,001)$, pasando de $10 \%$ en 1990 a $45 \%$ en 1996 y 58\% en 1997 (figura 2).

\section{Evolución de la resistencia en Shigella sonnei}

El aumento de la resistencia a la ampicilina no fue estadísticamente significativo (de 36 a $54 \% ; \chi_{1 \mathrm{gl}}^{2}=0,18 ; P=$ $0,20)$. Tampoco lo fue la disminución de la resistencia a la TMP-SMX (de 82 a $\left.55 \% ; \chi_{1 \mathrm{gl}}^{2}=1,1 ; P=0,08\right)$, posiblemente debido al pequeño tamaño de las muestras ( $n=11$, tanto en 1990 como en 1997). Con respecto al cloranfenicol, solo se encontraron dos aislados resistentes, uno en 1995 y otro en 1997 (figura 3). Dos aislados mostraron resistencia múltiple a la ampicilina, TMP-SMX y cloranfenicol.

\section{DISCUSIÓN}

Este estudio mostró una tendencia al aumento de la resistencia a los antimicrobianos más usados en nuestro medio en el tratamiento de la shigelosis en pediatría. Esto también se ha observado $(5,6)$ en otros países, como los Estados Unidos, Israel, Inglaterra y Gales, pero con porcentajes inferiores a los registrados en este estudio. En estos países la especie más frecuente fue $S$. sonnei, seguida de $S$. flexneri y $S$. boydii, mientras que en nuestra experiencia la más frecuente fue $S$. flexneri, seguida de S. sonnei y S. boydii. En países no industrializados, particularmente en los africanos, y también en Bangladesh, se ha registrado un elevado índice de resistencia (5), pero las especies que tuvieron mayor relevancia fueron $S$. flexneri y $S$. dysenteriae I.

En el presente trabajo $S$. flexneri fue significativamente más resistente que S. sonnei, principalmente a la ampicilina, al TMP-SMX y al cloranfenicol, y esto es de gran importancia ya que $S$. flexneri fue la especie aislada con mayor frecuencia en pacientes tanto ambulatorios como hospitalizados. No se apreciaron diferencias de resistencia entre los aislados obtenidos en uno y otro grupo de pacientes, posiblemente 
FIGURA 3. Porcentajes de aislados de Shigella sonnei resistentes a la ampicilina y al trimetoprima-sulfametoxazol

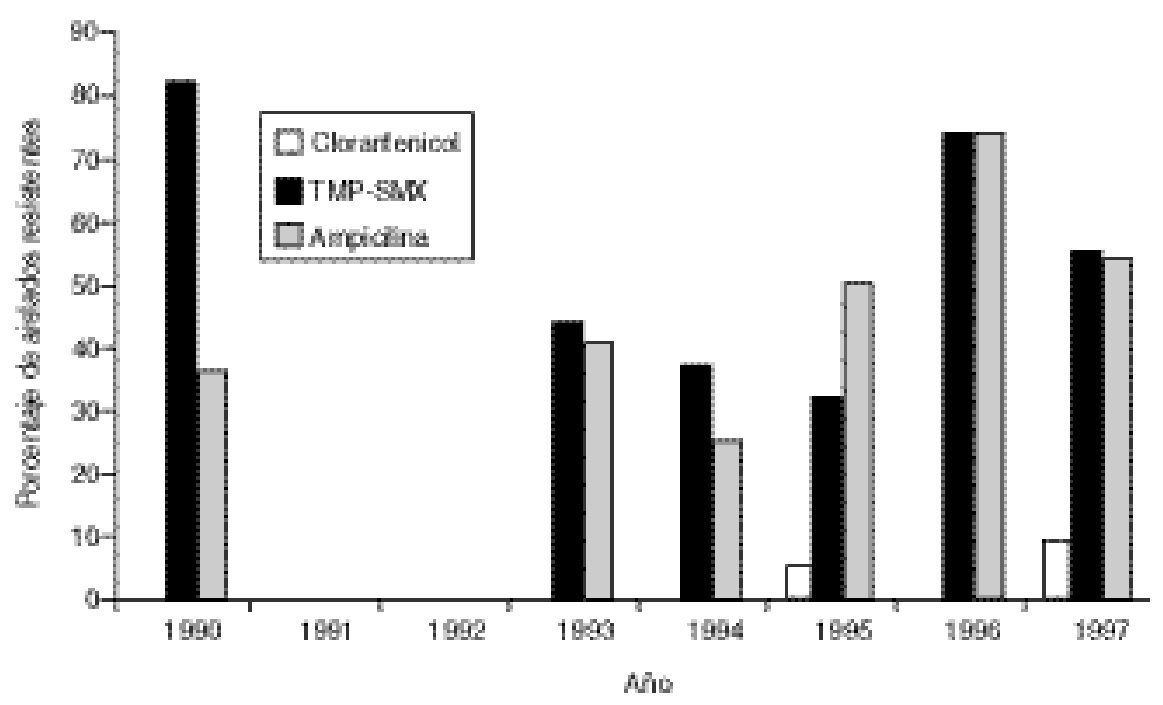

debido a la presión de selección ejercida por antimicrobianos de uso común en pacientes ambulatorios (10), lo cual se ha observado también en otros estudios (5).

En los países desarrollados se postulan (6) como origen de la resistencia de Shigella, y particularmente de $S$. flexneri, los viajes a países no industrializados. Es preocupante, además, el aumento de la resistencia múltiple a la ampicilina, al TMP-SMX y al cloranfenicol en nuestros aislados de $S$. flexneri, ya que, tratándose de pacientes pediátricos, las posibilidades terapéuticas se ven restringidas, pues los agentes efectivos frente a la shigelosis (1), tales como las quinolonas, no están aún autorizados en pediatría, debido al posible daño que causarían en el cartílago en desarrollo. En la experiencia clínica de nuestro hospital, una buena alternativa es la fosfomicina, que también ensayamos in vitro y que no ha mostrado resistencia hasta el momento. Otra alternativa podría ser la furazolidona, un antibiótico que ensayamos rutinariamente desde 1995 sin que tampoco hayamos registrado resistencia hasta el momento.

Ante lo expuesto y dado que las especies de Shigella y la resistencia varían según la localización geográfica, cobran gran importancia el control y seguimiento de los patrones de resistencia para la selección del tratamiento empírico adecuado en pacientes con shigelosis. Es obligatorio realizar pruebas de susceptibilidad en cada aislado clínico, ya que permiten detectar cambios en el perfil de la resistencia $y$, con ello, adecuar el tratamiento empírico.

\section{REFERENCIAS}

1. Ashkenazi S, Cleary T. Shigella infections. En: Feigin RD, Cherry JD, eds.Textbook of pediatric infectious diseases. Philadelphia: Saunders; 1992. pp. 637-646.

2. Gilligam PH, Janda M, Karmali M, Miller M. Laboratory diagnosis of bacterial diarrhea. Ed. Board for ASM Cumitechs. Cumitech 12, 1980:1-25.

3. Report of Committee on Infectious Diseases. 22a. ed. Elk Grove Village, IL: American Academy of Pediatrics; 1991. pp. 427-429.

4. Bishop WP, Ulshem MH. Bacterial gastroenteritis. En: Lebenthal E, ed. Pediatric Gastroenterology I. Philadelphia: Saunders; 1988. pp. 75-77.

5. Ashkenazi S, May-Zahav M, Sulkes J, Zilberberg R, Samra Z. Increasing antimicrobial resistance of Shigella isolates in Israel during the period 1984 to 1992. Antimicrobial Agents and Chemotherapy 1995;39:819-823
6. Cheasty T, Skinner JA, Rowe B, Threlfall EJ. Increasing incidence of antibiotic resistance in Shigellas from humans in England and Wales: recommendations for therapy. Microbial Drug Resistance 1998;4:57-60.

7. Hoge CW, Gambel JM, Srijan A, Pitarangsi C, Echeverria P. Trends in antibiotic resistance among diarrheal pathogens isolated in Thailand over 15 years. Clin Infect Dis 1998;26: 341-345.

8. Kelly MT, Brenner J, Farmer III JJ. Enterobacteriaceae. En: Ballows A, Hausler WJ, Shadomy HJ, eds. Manual of Clinical Microbiology. 4a. ed. Washington D.C.: American Society for Microbiology; 1995. pp. 347-352.

9. National Committee for Clinical Laboratory Standards. Performance standards for antimicrobial susceptibility testing: Third informational supplement NCCLS. Document M2-A5 Villanova, PA: National Committee for Clini- cal Laboratory Standards. Approved Standard 1993.

10. Dowell SF, Marcy SM, Phillips WR, Gerber MA. Principles of judicious use of antimicrobial agents for pediatric upper respiratory tract infections. Pediatrics 1998;101:163-165.

Manuscrito recibido el 11 de septiembre de 1998 y aceptado para publicación, tras revisión, el 27 de agosto de 1999. 
ABSTRACT This study analyzed the evolution of antimicrobial resistance in 771 isolates of Shigella spp. obtained from a total of 9195 feces cultures done between 1990 and 1997 in a children's hospital in Córdoba, Argentina. S. flexneri, which was responsible for $73 \%$ of the Shigella infections, was the species with the greatest resistance. The frequency of S. flexneri resistance to the three antibiotics most used (ampicillin, trimethoprimsulfamethoxazole, and chloramphenicol) increased from 10\% in 1990 to 58\% in 1997 $(P<0.001)$. Considering each of the drugs individually, the resistance to ampicillin increased from $60 \%$ to $100 \%(P<0.001)$, the resistance to chloramphenicol from $13 \%$ to $71 \%(P<0.001)$, and the resistance to trimethoprim-sulfamethoxazole from $79 \%$ to $84 \%(P=0.22)$. For $S$. sonnei, the increase in resistance to ampicillin (from $36 \%$ in 1990 to $54 \%$ in 1997) was not statistically significant $(P=0.20)$, nor was the reduction in resistance to trimethoprim-sulfamethoxazole, which went from $82 \%$ in 1990 to $55 \%$ in $1997(P=0.08)$. Only two $S$. sonnei isolates were found that were resistant to chloramphenicol, one in 1995 and another in 1997; two S. sonnei isolates were found with resistance to all three antibiotics. We consider it essential to carry out susceptibility tests of each Shigella clinical isolate, to detect changes in the resistance profile and thus modify empiric treatment. 